\title{
The Use of Whole Blood Transfusion in Trauma
}

\author{
Mary Hanna ${ }^{1}$ · Justin Knittel ${ }^{2}$ · Jason Gillihan ${ }^{2}$ (]
}

Accepted: 10 November 2021 / Published online: 17 January 2022

(c) The Author(s), under exclusive licence to Springer Science+Business Media, LLC, part of Springer Nature 2022

\begin{abstract}
Purpose of Review This review illustrates the current benefits, limitations, ongoing research, and future paths for Low Titer O Whole Blood compared to Component Therapy in massive transfusion for trauma patients.

Recent Findings Many studies show that compared to Component Therapy, Low Titer O Whole Blood transfusion is associated with better patient outcomes and simplified transfusion logistics among others. There are, however, issues with cost, supply/demand and handling of Whole Blood that limit its use, but experience in the military setting has shown that these limitations can be easily overcome.

Summary The use of Whole Blood has increased in the civilian trauma population and there is a growing body of evidence to support its current use. More research looking at Whole Blood in females of child-bearing age, pediatric populations, and cold-stored platelets is underway.
\end{abstract}

Keywords Damage control resuscitation (DCR) $\cdot$ Low Titer O Whole Blood (LTOWB) · Massive transfusion protocol (MTP) $\cdot$ Component therapy $(\mathrm{CT}) \cdot$ Whole blood (WB)

\section{Introduction}

Trauma is the fourth leading cause of mortality in the USA. More than $30 \%$ of trauma-related deaths are due to massive hemorrhage $[1 \bullet]$. Furthermore, greater than $90 \%$ of potentially preventable battlefield deaths occur in the prehospital setting and are a result of hemorrhage. The majority of these are from noncompressible torso hemorrhage [2]. This poses a significant issue for the health care system as these deaths may be preventable with adequate resuscitation. Damage control resuscitation (DCR) is a systematic approach to the management of the trauma patient, which aims to limit blood loss and prevent coagulopathy starting in the pre-hospital

This article is part of the Topical Collection on Anesthesia for Trauma

Jason Gillihan gillihaj@wustl.edu

1 Department of Anesthesiology and Pain Medicine, University of Ottawa, The Ottawa Hospital, Ottawa, ON, CA K1S5W1, Canada

2 Department of Anesthesiology, Washington University School of Medicine, Campus Box 8054, 600 S Euclid Ave, St Louis, MO 63110, USA setting with early blood transfusion and massive transfusion protocols (MTP).

The practice of using whole blood (WB) by the US Army Medical Department dates to World War I and continued through World War II, the Korean War and the Vietnam War. In the 1960's and the 1970's, however, blood banking capabilities became more advanced and the ability to process and store blood products made component therapy (CT) the preferred method of transfusion. Thus, CT with packed red blood cells (RBCs), fresh frozen plasma (FFP), platelets (PLTs) and cryoprecipitate replaced WB as the preferred treatment of hemorrhagic shock in the military and civilian population, despite a lack of evidence to support this shift [3].

There is a growing amount of evidence today that proves that a balanced resuscitation with roughly equal ratio of units of packed red blood cells, fresh frozen plasma, and pooled platelets leads to better outcomes in massive transfusion resuscitation and prevents acute traumatic coagulopathy in DCR [3]. Because of the growing evidence that supports balanced resuscitation, there is a renewed interest in the use of WB in military care setting as part of DCR. As such, in 2014 the Committee on Tactical Combat Casualty Care recommended WB as the optimal resuscitation product on the battlefield and prehospital combat theaters 
[4]. More specifically, the American Association of Blood Banks (AABB) endorses the use of Low Titer Type O Whole Blood (LTOWB) as universal donor WB. There is growing evidence that supports the use of LTWOB over CT in massive transfusion, but more high-quality studies are needed. The purpose of this article is to compare benefits, limitations and future paths between the use of WB and CT.

\section{Description of Whole Blood Compared to Component Therapy}

Currently, in the military care setting, the Armed Services Blood Program (ASBP) sends approximately 200 units of LTOWB weekly to support combat operations [5]. WB is collected in citrate-phosphate dextrose (CPD) storage solution and stored between $1{ }^{\circ} \mathrm{C}$ and $6{ }^{\circ} \mathrm{C}$. WB can last up to 21 days in CPD and up to 35 days in citrate-phosphate dextrose-adenine (CPDA) solution. Most centers in the USA limit the use of WB to 14-21 days, and the product does not need to be agitated or frozen. Due to the risk of transfusion related acute lung injury (TRALI), the majority of institutions utilize group $\mathrm{O}$ Rhesus $(\mathrm{Rh})$ positive male donors for all males, and females over the age of 50. Group O Rh negative WB is typically reserved for females of child-bearing age. LTOWB is group O WB with low levels of anti-A and anti-B immunoglobulins $\mathrm{M}(\operatorname{IgM})$, defined as a titer of less than 1:256. Some centers have a higher threshold for titers; these levels can reach as low as 1:50. These immunoglobulins occur as both $\operatorname{IgG}$ and $\operatorname{IgM}$, with IgM being the greatest concern to cause immediate transfusion reaction [3].

One unit of WB typically contains $500 \mathrm{~mL}$ and is composed of a hematocrit of $38-50 \%$, platelet count of $150-400 \mathrm{~K}, 100 \%$ plasma coagulation factors and $1000 \mathrm{mg}$ of fibrinogen. As Table 1 demonstrates, CT has a larger volume and does not attain the same level of concentrated blood products and coagulation activity as WB [6]. In fact, one of each traditional unit of pRBC, PLTs, FFP and cryoprecipitate equates to approximately $675 \mathrm{~mL}$ of blood products. In reality, we use 4-5 units of pooled random donor platelets, which then equates to the physiological platelet level in WB, as such the volume of $\mathrm{CT}$ is greatly underestimated when compared to WB. Furthermore, one unit of pRBCs has a shelf life of 35 days and is usually stored in a refrigerator between $1{ }^{\circ} \mathrm{C}$ and $6{ }^{\circ} \mathrm{C}$. One unit of PLTs requires an agitator and is stored at room temperature, ideally between $20^{\circ} \mathrm{C}$ and $24^{\circ} \mathrm{C}$. PLTs are generally acceptable to use for 5 days only. FFP and cryoprecipitate require temperatures of less than $-18^{\circ} \mathrm{C}$ and have a shelf-life of 1 year.

\section{Comparison Between Whole Blood and Component Therapy}

\section{Benefits}

As described in the previous section, the use of WB is associated with less processing, as there is no need for a centrifuge machine to separate the components, which also limits the waste of components due to the separation process. In addition, bacterial contamination risk is limited since WB can be refrigerated. Whole blood uses less additive compared to CT. For example, using 6 units of LTOWB has approximately $378 \mathrm{~mL}$ of preservative solution, whereas using 6 units of pRBC +6 units of FFP +1 unit of PLTs has $1055 \mathrm{~mL}$ of this solution. Therefore, less overall volume is given in WB MTP compared to CT, which then reduces the risk of trauma induced coagulopathy (TIC.) In fact, a study examining the hemostatic profiles of WB compared to CT by rotational thromboelastometry (ROTEM) demonstrated that compared to CT, WB was associated with a better hemostatic profile [3]. This is not surprising, given the fact that WB contains all coagulation factors and roughly $1000 \mathrm{mg}$ of fibrinogen.

Furthermore, there may be some benefit in delivering cold-stored products as part of WB resuscitation versus component therapy. For example, cold-stored PLTs seem to be functionally superior to room temperature stored PLTs even with the use of a leukoreduction filter [ $7 \bullet \bullet]$. Historically, there has been concern about the viability of cold stored PLTs after transfusion due to molecular changes to platelet receptors leading to removal from circulation by

Table 1 Whole blood composition compared to component therapy

\begin{tabular}{ll}
\hline Component therapy $(675 \mathrm{~mL})$ & Whole blood $(500 \mathrm{~mL})$ \\
\hline 1 unit of $\mathrm{pRBC}=335 \mathrm{~mL}$ with hematocrit of $55 \%$ & Hematocrit of $38-50 \%$ \\
1 unit of PLTs $=50 \mathrm{~mL}$ with $88 \mathrm{~K}$ platelets & Platelet count of $150-400 \mathrm{~K}$ \\
1 unit of FFP $=275 \mathrm{~mL}$ with $80 \%$ coagulation activity & Plasma coagulation factors $=100 \%$ \\
1 unit of cryoprecipitate $=15 \mathrm{~mL}$ with $150 \mathrm{mg}$ of fibrinogen & Fibrinogen $=1000 \mathrm{mg}$ \\
$\begin{array}{l}\text { Thus, } 1 \text { unit of pRBC }+1 \text { unit of PLTs }+1 \text { unit of FFP }+1 \text { unit of cryoprecipitate }=675 \mathrm{~mL} \text { with hematocrit of } 29 \% \text {, platelet count of } 88 \mathrm{~K} \text { and } \\
\text { coagulation activity of } 65 \% \text { compared with WB }\end{array}$ \\
\hline
\end{tabular}

$p R B C$ packed red blood cells, $P L T$ s platelets, $F F P$ fresh frozen plasma, $W B$ whole blood. 
macrophages within a few hours of transfusion. Early studies in cancer patients with thrombocytopenia showed that patients who received platelets stored at room temperature had greater increases in platelet counts for a longer duration of time [8]. However, in vitro studies suggest that cold stored PLTs are more hemostatically active once in circulation, even if they are cleared at a faster rate. With the goal of hemostasis in the setting of acute bleeding, circulation time may factor less than functionality. The same work has demonstrated that maximum clot strength was within acceptable range on day 35 of cold-stored WB when measured by thromboelastography $[7 \bullet \bullet]$.

Each unit of CT pRBC contains less than $10 \mathrm{~mL}$ of plasma and as such, type O pRBC can be used for transfusion regardless of $\mathrm{ABO}$ blood group of the recipient. Traditionally, CT with FFP and platelets need to be ABO compatible due to the risk of transfusion-related immunological reactions [9]. WB contains plasma and as discussed previously; the amount is almost equal to the amount contained in a traditional unit of FFP. This may result in clinically relevant direct intravascular hemolysis of the transfused RBCs in the recipient, depending on the quantity of antibodies present in the unit. Despite this fact, there is significant evidence that demonstrates that this is not the case, and that the risk of intravascular hemolysis is not any different or even any higher than with CT. Currently, most centers use LTOWB with low levels of anti-A and anti-B IgM defined as a titer of less than 1:256, although there is no definition of a volume of incompatible plasma that can be safely used without consequence of hemolysis [9]. In fact, there is evidence that transfusion with WB with anti-A and anti-B titers as high as 400 was not related to major transfusion-related reactions [10]. There are many advantages of giving WB when compared to CT in terms of patient outcomes. In 2020 Hanna et al. examined 8900 patients who received transfusions of WB or CT [1•]. On regression analysis, they demonstrated that the WB was independently associated with reduced 24-h mortality (odds ratio [OR], 0.78 [0.59-0.89]; $p=0.006$ ), in-hospital mortality (OR, 0.88 [0.81-0.90]; $p=0.011)$, and major complications such as acute kidney injury (AKI), acute respiratory distress syndrome (ARDS), deep vein thrombosis (DVT) and pulmonary embolism (PE) (OR, 0.92 [0.87-0.96]; $p=0.013$ ).

Furthermore, this study also demonstrated a statistically significant shorter length of hospital stay with WB transfusion ( 9 days vs 15 days with CT). A study by Spinella et al. also demonstrated that the use of WB was associated with an improved 30-day survival (OR 12.4; $p=0.01$ ) [11]. Interestingly, the combination of WB with some elements of CT (pRBCs and FFP) led to better outcomes than using $\mathrm{CT}$ alone. This was shown by Nessen et al., where transfusing WB with some element of CT led to lower mortality (OR, 0.096; $p<0.01$ ) than using CT only [12]. Finally,
Williams et al. demonstrated that WB is associated with a $53 \%$ reduction in post-emergency department blood product transfusion (OR 0.47; 95\% CI, 0.23-0.94, $p=0.033$ ) and two-fold increase in likelihood of survival (OR 2.19; $95 \%$ CI, 1.01-4.76; $p=0.047$ ) [13].

In terms of logistics, WB has many advantages over CT, especially during massive resuscitation. WB can be given through a warmed rapid infuser during MTP whereas this is not recommended for all elements of CT. Currently, it is recommended that PLTs in CT should be given at room temperature. Commonly, this means separate intravenous (IV) access and lines for platelet transfusion. During MTP, this is one less thing that needs to be thought about as WB arrives in a cooler and can be administered rapidly in the same IV access. With CT, there has to be time given to setting up a different line for PLTs and ensuring that these are not given through the warmer. Also, logistically, WB is sent right away and does not need to be thawed, like FFP and cryoprecipitate do. In MTP, this is time that can be allotted to managing other aspects of the patient's care.

Furthermore, there is a decreased likelihood of an administrative error with WB transfusion when compared to CT, which is more likely to result in severe hemolysis. Because $\mathrm{CT}$ is a combination of multiple blood products that each must be checked against the patient blood type, the likelihood of a clerical error and mismatch increases. Since WB is only Low Titer Type O Blood, there is a much lower risk of such an error [2].

\section{Limitations}

Despite many of the benefits of the use of LTOWB in trauma, there are some limitations that complicate its use. To begin, initiating a WB program in a blood bank can be very expensive as this cost is added onto the already established CT branch of the hospital's blood bank. For example, at Barnes Jewish Hospital, in association with Washington University School of Medicine in St. Louis, there is an average of 150 MTP activations for Level 1 trauma patients annually. The institution of a WB program for this number of MTP activations added an estimated additional \$170,000 USD in costs of blood products annually. This cost addition is on-par with most US centers who have also added WB to their blood bank inventory. Due to uncertain benefits and high cost, many blood banks or hospital administrators may challenge the introduction of LTOWB. This underscores the importance of performing additional high quality Randomized Control Trials (RCTs) to prove the benefit of WB use in major trauma.

Another major challenge for the implementation of a WB program in a major trauma center is the logistical constraints of shipping, handling and cold chain management [14]. The 
military has overcome many of the challenges of maintaining blood products within tight parameters, especially in austere environments, such as developing progressively smaller and lighter blood storage containers to help medics carry blood products in their aid bags to ensure blood transfusions are available without delay. The military experience is proof that civilian blood bank programs may also surmount the logistical constraints of a WB branch in their blood bank.

In "normal" times, supply/demand issues have always been a point of hardship for blood banks. Since the beginning of the COVID-19 pandemic, blood donations have declined nationally. This has led to significant disruptions in supply of all blood components, including LTOWB. As we learn to navigate this new situation, we hope that the number of blood donations continues to increase and help replenish the LTOWB supply. The other issue with supply is finding the right candidates for LTOWB donations. At the moment, Group $\mathrm{O} \mathrm{Rh}+$ males are the ideal donors to mitigate the risk of TRALI. This significantly reduces the pool of donors. Another significant limitation is the practice of excluding donors who have had aspirin within $48 \mathrm{~h}$ of donation [15]. However, there is currently evidence in the literature [16, 17•] demonstrating that group A plasma can be safely given to patients with unknown blood type without any major risk of complications or increase in mortality. One may hope that this evidence may be extrapolated for the use of other WB blood types in trauma.

The final limitation is the issue of waste. Many US programs only validate LTOWB for 14 days of storage, meaning that there is a significant risk of blood wastage if there is an inability to match supply/demand, or if LTOWB cannot be easily reallocated to other uses, such as in emergency general surgery cases. Some centers are able to take WB on day 15 and centrifuge the unit, salvaging the pRBC's, to be used again by the blood bank, but unfortunately, not all hospitals adopt this measure. There are health systems, however, that have succeeded in implementing LTOWB with minimal waste. One such example is the San Antonio, Texas area, where the use of LTOWB by ground and helicopter EMS, outlying hospitals, and level 1 trauma centers was implemented with less than $1 \%$ blood waste [18].

\section{Future Paths}

\section{Childbearing Age Females and Rh Status}

As it currently stands, finding adequate number of $\mathrm{O}$ negative donors is challenging, as approximately $7 \%$ of the US population is $\mathrm{O}$ negative. Furthermore, there is an approximate $2-5 \%$ loss of eligible $\mathrm{O}$ negative donors due to restricting the ABO titer cutoff to less than 1:256. Finally, there is even greater loss as the donor pool is further restricted by limiting donor base to males and never pregnant females. The pool of suitable donors is therefore reduced down to less than $5 \%$ of the overall donor base [18]. Especially during the COVID-19 pandemic, blood donations have been limited and it is even harder to find males who are $\mathrm{O}$ negative. A potential solution is to allow O positive WB transfusion to females of child-bearing age, who require MTP. For the female to have alloimmunization, she must make anti-D antibodies, must survive MTP in trauma, must then become pregnant in the future and her fetus must be $\mathrm{D}+$. A gravid patient with anti-D antibodies with a $\mathrm{D}+$ fetus has a rate of fetal demise of approximately $4 \%$, whereas the risk of one dying from trauma without transfusion is significantly higher. The benefits in this case outweigh the risks and it seems reasonable to allow females of child-bearing age to be recipient of O positive WB. Additional research is needed in this area to elucidate whether this is feasible and safe.

\section{Pediatrics}

The first cohort of pediatric civilian trauma patients to receive WB during resuscitation was described in a study by Children's Hospital of Pittsburgh of the University of Pittsburgh Medical Center in 2018 [19]. Based on the preliminary data, $20 \mathrm{ml} / \mathrm{kg}$ of WB was safe to use in pediatric patients. This was again studied in 2020 by the same center with $40 \mathrm{ml} / \mathrm{kg}$ of WB used in children. In this study, WB transfusion resulted in faster resolution of shock, lower posttransfusion INR, and reduced need for CT product transfusion [20].

More recently, another study demonstrated WB transfusion resulted in significantly decreased total blood products transfused both at $4 \mathrm{~h}(35$ [22-73] vs. 48 [33-95] mL/kg; $p=0.013)$ and $24 \mathrm{~h}(39$ [24-97] vs. 53 [36-119] mL/kg; $p<0.001)$. Mortality rate at $24 \mathrm{~h}(19.3 \mathrm{vs.} 21.9 \% ; p=0.546)$ and in-hospital mortality (31.1 vs. $34.4 \% ; p=0.502)$ were not different. Similarly, no difference in hospital length of stay (LOS) and rates of major complications were found. Patients in the WB group required significantly less ventilation days (2 [2-6] vs. 3 [2-8] days; $p=0.021)$ [21]. Although this new evidence is very promising, more studies are currently required and are in progress looking at WB transfusion in pediatrics.

\section{Research}

There are a multitude of new trials being carried out evaluating the benefits of LTOWB. A few are mentioned in this discussion. Washington University in St. Louis in association 
with the University of Minnesota Medical School in Minneapolis received 26 million USD in funding to determine whether cold-stored platelets can reduce blood loss more effectively than those stored at room temperature. The PPOWER (Pragmatic Prehospital, Type O, Whole Blood Early Resuscitation) trial is a single center, 3-year prospective randomized pilot clinical trial with the experimental group receiving LTOWB in the prehospital and hospital setting followed by standard CT resuscitation compared with standard CT resuscitation. The STORHM study (Sang TOtal pour la Reanimation des Hemorragies Massive) is a French study looking at WB in traumatic massive hemorrhage. This non-inferiority study will compare LTOWB compared to 1:1:1 fixed ratio with endpoints including mortality, thromboelastography (TEG) parameters, multi-organ failure and lactate clearance. Finally, another study is the SWAT trial (Shock, Whole Blood and Assessment of TBI). This 4-year, multicenter, prospective observational cohort study will trial WB compared to standard CT.

\section{Conclusion}

LTOWB in trauma MTP has become common practice in over 70 civilian centers in the USA [17•]. Furthermore, other settings such as cardiac surgery, liver transplant and obstetrical hemorrhage are beginning to follow suit. Compared to CT, WB offers many benefits, such as reduced volume transfused (leading to decreased TIC), reduced 24-h mortality, simpler transfusion logistics, and better overall outcomes in trauma patients. There are many limitations with the implementation of WB therapy, such as cost, supply/demand, shipping, and handling. Despite these limitations, there are many hospitals across the USA that are adding WB to their blood banks and finding methods and researching ways to overcome these obstacles.

Many questions still need to addressed, such as the use of WB in pediatrics, benefit of cold-stored platelets, and adequate titer level for safe transfusion. Overall, WB has become a standard of practice in the military setting, and this experience shows many promising benefits to be adapted in the civilian population.

\section{Declarations}

Conflict of Interest The authors do not have any potential conflicts of interest to disclose.

\section{References}

Papers of particular interest, published recently, have been highlighted as:

- Of importance

$\bullet$ Of major importance

1. Hanna K, Bible L, Chehab M, Asmar S, et al. Nationwide analysis of whole blood hemostatic resuscitation in civilian trauma. J Trauma Acute Care Surg 2020; 89 (2):329-335. An important paper given that the majority of U.S. programs have opted for introduction of WB for trauma resuscitations, but still rely heavily on CT (either due to supply issues associated with WB, or by design in their MTP protocol). This paper specifically looks at outcomes of WB as an adjunct to $\mathrm{CT}$ resuscitation in trauma, which reflects current practice in many Level-1 trauma centers.

2. Eastridge BJ, Mabry RL, Sequin P, et al. Death on the Battlefield (2001-2011): implications for the future of combat casualty care. J Trauma Acute Care Surg. 2012;73(6 Suppl 5):S431-7.

3. Kornblith LZ, Howard BM, Cheung CK, et al. The whole is greater than the sum of its parts: hemostatic profiles of whole blood variants. J Trauma Acute Care Surg. 2014;77(6):818-27.

4. Butler FK, Holcomb JB, Schreiber MA, et al. Fluid resuscitation for hemorrhagic shock in tactical combat casualty care: TCCC guidelines change 14-01--2 Jun 2014. J Spec Oper Med. 2014;14(3):13-38.

5. Butler F, Holcomb JB, Shackelford S, et al. Advanced resuscitative care in tactical combat casualty care: TCCC guidelines change 18-01:14 October 2018. J Spec Oper Med. 2019;18(4):37-55.

6. Sihler et al. Complications of Massive Transfusion. Chest. 2010; 137(1):209-220

7.• Sivertsen J, Braathen H, Felli Lunde TH, Kristoffersen EK, et al. Coldstored leukoreduced CPDA-1 whole blood: in vitro quality and hemostatic properties. Transfusion. 2020; 60; 1042-1049. A very important paper that explores the hemostatic properties of cold-stored WB, which is currently the focus of several research projects. This paper helps to inform the decision around "shelf-life" of LTOWB in your local blood bank. It also relates to current research on hemostatic properties of cold platelets vs. traditional, room temperature stored platelets.

8. Djerassi I, Farber S. Conference on obstacles to the control of acute leukemia. Control and prevention of hemorrhage: platelet transfusion. Cancer Res. 1965;25(9):1499-503.

9. Harm et al. Immunologic risk of whole blood: ABO compatibility, D alloimmunization, and transfusion-related acute lung injury. Transfusion. 2019;59;1507-1511

10. Berseus et al. Risks of hemolysis due to anti-A and anti-B caused by the transfusion of blood or blood components containing ABO-incompatible plasma. Transfusion. 2013; 53:114S-123S

11. Spinella PC, Perkins JG, Grathwohl KW, et al. Warm fresh whole blood is independently associated with improved survival for patients with combat-related traumatic injuries. J Trauma. 2009;66:S69-76.

12. Nessen SC, Eastridge BJ, Cronk D, Craig RM, et al. Fresh whole blood use by forward surgical teams in Afghanistan is associated with improved survival compared to component therapy without platelets. Transfusion. 2013;53:107S-113S.

13. Williams J, Merutka N, Meyer D, Bai Y, Prater S, Cabrera R, Holcomb JB, Wade CE, Love JD, Cotton BA. Safety profile and 
impact of low-titer group $\mathrm{O}$ whole blood for emergency use in trauma. J Trauma Acute Care Surg. 2020;88(1):87-93.

14. Fisher AD, Miles EA, Broussard MA, et al. Low titer group O whole blood resuscitation: Military experience from the point of injury. J Trauma Acute Care Surg. 2020;89(4):834-40.

15. Troughton M, Young P. Conservation of Rh negative Low Titer $\mathrm{O}$ Whole Blood (LTOWB) and the need for a national conversation to define its use in trauma transfusion protocols. Transfusion. 2021;61:1966-71.

16. Dunbar N, Yazer M. Safety of the use of group A plasma in trauma: the STAT study. Transfusion. 2017;57:1879-84.

17. Seheult, J. Dunbar, N. Transfusion of blood components containing ABO-incompatible plasma does not lead to higher mortality in civilian trauma patients. Transfusion. 2020; 60(11): 25172528. An important paper that speaks to the concern held by many regarding transfusion of $\mathrm{ABO}$-incompatible plasma, which weighs in on institutional decisions around "caps" on numbers of units of LTOWB used for MTP protocols.
18. Zhu CS, Pokorny DM, Eastridge BJ, et al. Give the trauma patient what they bleed, when and where they need it: establishing a comprehensive regional system of resuscitation based on patient need utilizing cold-stored, low-titer $\mathrm{O}+$ whole blood. Transfusion. 2019;59(S2):1429-38.

19. Leeper C, Yazer M, et al. Use of Uncrossmatched Cold-Stored Whole Blood in Injured Children With Hemorrhagic Shock. JAMA Pediatr. 2018;172(5):491-2.

20. Leeper C, Yazer M, et al. Whole Blood is Superior to Component Transfusion for Injured Children: A Propensity Matched Analysis. Ann Surg. 2020;272(4):590-4.

21. Anand, T. Obaid, O. et 1. Whole Blood Hemostatic Resuscitation in Pediatric Trauma: A Nationwide Propensity-Matched Analysis. J Trauma Acute Care Surg. 2021: Online ahead of print.

Publisher's Note Springer Nature remains neutral with regard to jurisdictional claims in published maps and institutional affiliations. 